\title{
Value of MR Imaging in Differentiation between Solitary Fibrous Tumor and Schwannoma in the Orbit
}

\author{
Z. Zhang, J. Shi, J. Guo, F. Yan, L. Fu, and J. Xian
}

\begin{abstract}
BACKGROUND AND PURPOSE: Orbital SFT is a rare tumor, often misdiagnosed as orbital schwannoma preoperatively but with different prognosis and treatment. Our aim was to evaluate MR imaging features that might distinguish orbital SFT from schwannoma.
\end{abstract}

MATERIALS AND METHODS: MR imaging including DCE scanning was performed in 9 patients with SFT and 22 patients with schwannoma in the orbit confirmed by pathology. Location, shape, margin, signal intensity, homogeneity, enhancement pattern, ER, and TIC of the tumors were retrospectively evaluated.

RESULTS: There was a statistically significant difference between SFT and schwannoma in location and T2 signal intensity $(P<.05)$. A statistically significant difference was also found regarding the enhancement pattern of the very high-signal-intensity areas shown on T2-weighted imaging and the type of TICs $(P<.01)$.

CONCLUSIONS: MR imaging is useful in differentiating orbital SFT and schwannoma. The enhancement pattern of the very high-signalintensity areas shown on T2-weighted imaging and the type of TICs on DCE MR imaging played an important role in differentiating orbital SFT from schwannoma.

ABBREVIATIONS: $\mathrm{Cl}=$ contrast index; $\mathrm{ER}=$ enhancement ratio; $\mathrm{DCE}=$ dynamic contrast-enhanced; $\mathrm{SFT}=$ solitary fibrous tumor; TIC = time-intensity curve

$\mathbf{S}^{\mathrm{H}}$ FT, a spindle cell neoplasm arising from mesenchymal structures, is a rare neoplasm found in many locations including the orbit. ${ }^{1,2}$ Orbital solitary fibrous tumor was first recognized in 1994, and its histopathology has since been well-described. ${ }^{1,3-6}$ SFT demonstrates great variability in its morphology and shares histologic features with other benign mesenchymal tumors of this area such as schwannoma and hemangiopericytoma. ${ }^{5}$ SFT demonstrates a strong level of diffuse immunoreactivity with a monoclonal antibody directed against the human hematopoietic progenitor cell antigen CD34; while most schwannomas and some of

Received May 24, 2012; accepted after revision July 28.

From the Departments of Radiology (Z.Z., J.G., F.Y., L.F., J.X.) and Eye Center (J.S.), Beijing Tongren Hospital, Capital Medical University, Beijing, China.

Zhengyu Zhang and Jitong Shi shared equally in the authorship of this article and are co-first authors.

This work was in part supported by a grant (2010D003034000033) from Beijing Excellent Talents Foundation, China; and a grant (7112030) from Beijing Municipal Natural Science Foundation, China

Please address correspondence to Junfang Xian, MD, Department of Radiology, Beijing Tongren Hospital, Capital Medical University, Beijing 100730, China; e-mail: cjr.xianjunfang@vip.163.com

- Indicates open access to non-subscribers at www.ajnr.org

三 Indicates article with supplemental on-line tables

http://dx.doi.org/10.3174/ajnr.A3340 the hemangiopericytomas also can be CD34-positive, the CD34 immunoreactivity is generally weak in intensity and focal in distribution compared with that in SFT. ${ }^{7}$

Despite the distinct histologic and immunohistochemical appearance of SFT, its imaging appearance in the orbit has been considered nonspecific and it has often been misdiagnosed as schwannoma. ${ }^{8,9}$ Nonetheless, the prognosis and treatment of SFT and schwannoma in the orbit are different, ${ }^{2,6,10-15}$ and distinguishing SFT and schwannoma in the orbit is important because SFT is a more aggressive lesion than schwannoma.

SFTs are richly vascular with many dilated vessels. ${ }^{1-6}$ In the imaging evaluation of SFT in the orbit, sonography typically shows some degree of vascularity and is useful in the diagnosis of this disease. ${ }^{16} \mathrm{CT}$, especially dual-phase CT, demonstrates rapid enhancement with early washout of contrast material from the SFT tumor. ${ }^{2}$ DCE MR imaging has shown SFT as a highly vascularized mass with a prominent vascular pedicle that displaces adjacent structures, and it is more specific than $\mathrm{CT}$ in the diagnosis of this tumor. ${ }^{17}$

MR imaging plays an important role in the diagnosis of schwannoma and other tumors in the orbit, such as cavernous hemangioma, pleomorphic adenoma of the lacrimal gland, and dermoid cyst. ${ }^{13,18-22}$ MR imaging findings of orbital SFT have 
been reported in only a few cases, however, and little is known regarding the value of MR imaging in differentiating orbital SFT and schwannoma. ${ }^{2,8,15,23,24}$ The aim of this study was to evaluate MR imaging features that distinguish SFT from schwannoma in the orbit.

\section{PATIENTS AND METHODS \\ Patients}

Nine patients with orbital SFT and 22 patients with orbital schwannoma, all confirmed by pathology, were enrolled from January 2006 to October 2011. Nine patients with orbital SFT included 2 men and 7 women, with a mean age of 49 years (range, 27-69 years). In 22 patients with orbital schwannoma, 9 patients were male and 13 were female, with a mean age of 44 years (range, 10-71 years). The most common initial symptom was painless insidious proptosis, which was found in 23 patients, including 7 with SFTs and 16 with schwannomas. Other symptoms included decreased vision and swelling of the eyelid. Two cases of SFT were recurrent postsurgery with no chemotherapy or radiation therapy.

Informed consent was obtained from all patients for performance of radiologic studies and anonymized analysis of clinical and radiologic data.

\section{MR Imaging}

Two different MR imaging scanners were used in the study, including a 1.5T Signa TwinSpeed scanner (GE Healthcare, Milwaukee, Wisconsin) and a 3T Signa HDxt scanner (GE Healthcare). Of 9 patients with SFT, 3 were examined on the $1.5 \mathrm{~T} \mathrm{MR}$ imaging scanner and 6, on the 3T scanner. Of 22 patients with schwannoma, 15 examinations were performed on the 1.5T MR scanner and 7, on the 3T scanner. An 8-channel head coil was used for all patients.

Axial FSE T1WI, axial FSE T2WI, and coronal FSE T1WI were performed in all 31 patients. The imaging parameters on the $1.5 \mathrm{~T}$ scanner were the following: T1WI: TR, $500 \mathrm{~ms}$; TE, $12 \mathrm{~ms}$; T2WI: TR, 4000 ms; TE, 120 ms; NEX, 2; matrix, $288 \times 224$; FOV, $18 \times$ $18 \mathrm{~cm}$; section thickness, $3 \mathrm{~mm}$; gap, $0.3 \mathrm{~mm}$. The parameters on the 3T scanner were the following: T1WI: TR, $400 \mathrm{~ms}$; TE, $10 \mathrm{~ms}$; T2WI: TR, 3000 ms; TE, 120 ms; NEX, 2; matrix, $384 \times 256$; FOV, $18 \times 18 \mathrm{~cm}$; section thickness, $3 \mathrm{~mm}$; gap, $0.3 \mathrm{~mm}$.

DCE imaging was performed with a fast-spoiled gradient recalled sequence. The imaging parameters on the Signa TwinSpeed 1.5T scanner were the following: TR/TE, 4.8/1.9 ms; flip angle, $15^{\circ}$; 1 excitation; and section thickness, $3.2 \mathrm{~mm}$ at 0 intervals. Twelve scans were obtained. For each scan, 12 sections were obtained at 13 seconds. The interval between the 2 scans was 12 seconds. Total scanning time was 288 seconds. The parameters on the Signa HDxt 3T scanner were the following: TR/TE, 8.4/4 ms; flip angle, $15^{\circ}$; 1 excitation; and section thickness, $3.2 \mathrm{~mm}$ at 0 intervals. Thirty-seven scans were obtained. For each scan, 15 sections were obtained at 9 seconds. There was no interval between the 2 scans. Total scanning time was 344 seconds. A power injector (Spectris Solaris; Medrad, Indianola, Pennsylvania) with an injection flow rate of $2 \mathrm{~mL} / \mathrm{s}$ was used. The acquisition of the dynamic imaging began concurrently with the initiation of the injection of $0.1 \mathrm{mmol}$ of gadopentetate dimeglumine (Magnevist; Schering, Berlin, Germany) per kilogram of body weight.

Axial, coronal, and oblique sagittal FSE T1-weighted imaging were completed after DCE MR imaging. The parameters were the same as those in the precontrast sequence. Fat suppression was used in axial T1-weighted imaging.

\section{Image Analysis}

Two experienced head and neck radiologists (with 17 and 10 years of experience) reviewed the MR imaging studies and reached a consensus. Location, shape, margin, signal intensity, homogeneity, and enhancement pattern of the tumors were assessed. The location was defined as the preseptal orbit or postseptal orbit; extraconal space; intraconal space or both extraconal and intraconal spaces; superior or inferior orbit; and nasal or temporal orbit. The shape was defined as regular or irregular. The margin was defined as smooth or lobulated. The homogeneity of the mass was defined as homogeneous or heterogeneous on T1- and T2weighted imaging. Furthermore, if the tumor was heterogeneous on T2-weighted imaging, the existence of very high signal intensity like a CSF area within the tumor was observed. On contrastenhanced MR imaging including DCE MR imaging, the enhancement pattern, ER, and type of TIC of the tumors were evaluated. The enhancement pattern was divided into homogeneous and heterogeneous. The enhancement pattern of the very high-signalintensity areas shown on T2-weighted imaging was divided into 4 types: no enhancement, enhancement degree lower than that in the other part of the tumor (mild enhancement), enhancement degree identical to that in the other part of the tumor (moderate enhancement), and enhancement degree higher than that in the other part of the tumor (marked enhancement).

DCE MR imaging was evaluated by using an AW 4.4 workstation (GE Healthcare). A region of interest was drawn manually on the dynamic imaging for the assessment of the enhancement kinetics. We chose the area that demonstrated the greatest degree of early enhancement so that the TIC could be generated. The region of interest typically was $8 \mathrm{~mm}^{2}$. The CI was calculated from CI = [signal intensity (postcontrast) - signal intensity (precontrast)] / signal intensity (precontrast). ER was defined as follows: [signal intensity (maximum) - signal intensity (precontrast)] / signal intensity (precontrast). The TICs were analyzed qualitatively as washout, persistent, or plateau-shaped curves.

\section{Statistical Analysis}

Frequency distribution of individual MR imaging features in the SFT was compared with that in schwannoma with $\chi^{2}$ tests. The Fisher exact test was performed when the sample size in the subgroups was deemed too small. An independent-samples $t$ test was used for the difference in ER values between the 2 diseases. A $P$ value $<.05$ was considered to represent a statistical difference, and a $P$ value $<.01$ was considered to represent a significant statistical difference.

\section{RESULTS}

On-line Tables 1 and 2 describe the frequency distribution of precontrast MR imaging features (On-line Table 1) and postcontrast MR imaging features (On-line Table 2). There was a signifi- 

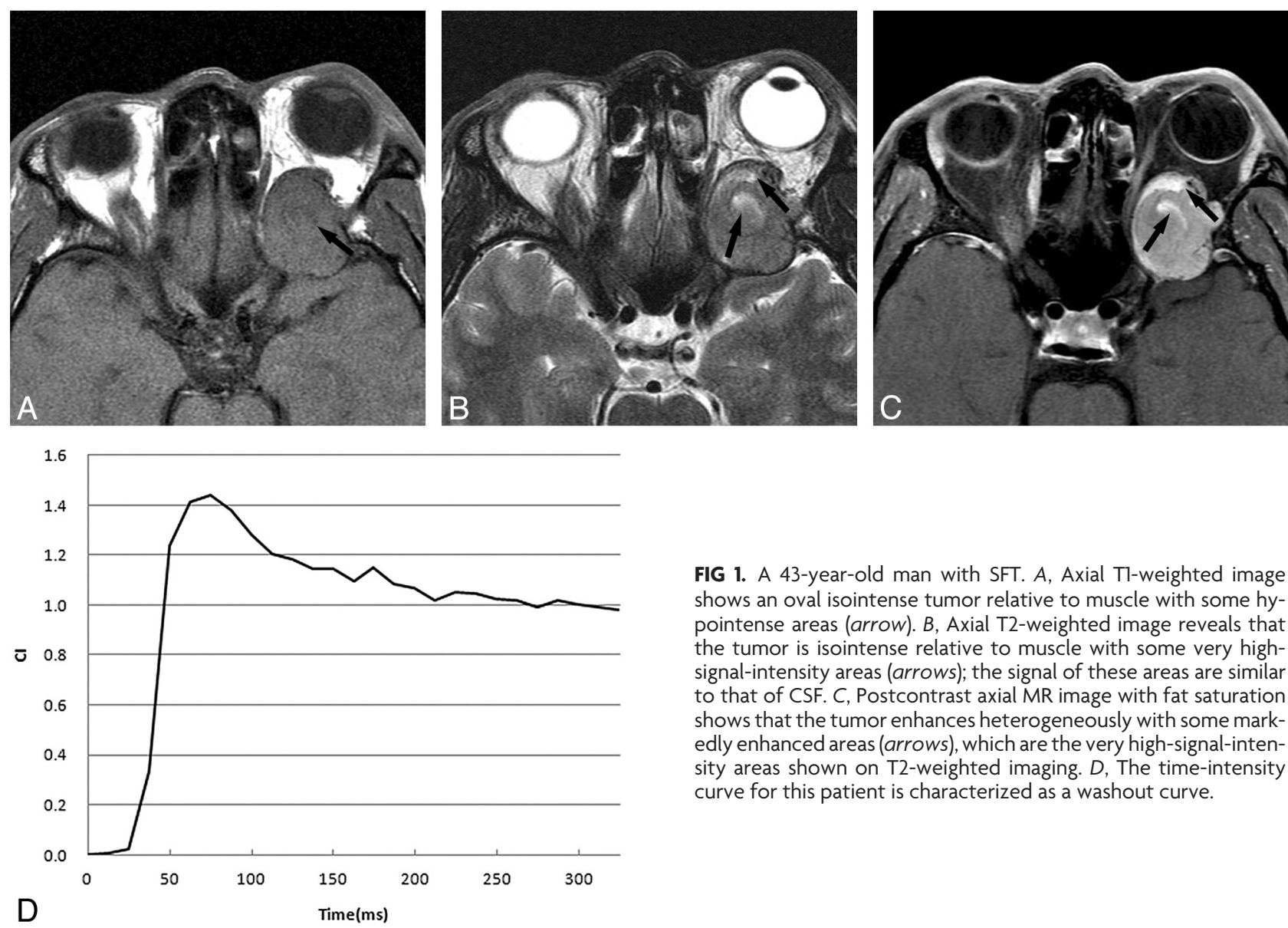

FIG 1. A 43-year-old man with SFT. A, Axial T7-weighted image shows an oval isointense tumor relative to muscle with some hypointense areas (arrow). B, Axial T2-weighted image reveals that the tumor is isointense relative to muscle with some very highsignal-intensity areas (arrows); the signal of these areas are similar to that of CSF. C, Postcontrast axial MR image with fat saturation shows that the tumor enhances heterogeneously with some markedly enhanced areas (arrows), which are the very high-signal-intensity areas shown on T2-weighted imaging. $D$, The time-intensity curve for this patient is characterized as a washout curve.

cant statistical difference between orbital SFT and schwannoma in the type of TICs and enhancement pattern of the very high-signalintensity areas shown on T2-weighted imaging $(P<.01)$. There was a statistical difference in the location (superior or inferior orbit) and T2 signal intensity between orbital SFT and schwannoma $(P<.05)$ (Figs 1 and 2$)$.

Orbital SFTs were always located in the superior portion of the orbit. They were usually isointense on T2-weighted imaging with washout TICs after contrast administration. Marked enhancement was most common in the very high-signal-intensity areas shown on T2-weighted imaging in SFT. Schwannomas more typically presented as hyperintense masses on T2-weighted imaging, located in the inferior or superior portion of the orbit with persistent or plateau-shaped TICs. The very high-signal-intensity areas shown on T2-weighted imaging usually exhibited no enhancement in schwannoma.

\section{DISCUSSION}

SFT most often arises from the pleura but can also arise from extrapleural sites such as lung, liver, breast, meninges, pelvis, sinonasal cavity, salivary gland, and orbit. ${ }^{1-8,11,23-26}$ Pathologically, orbital SFT is a well-defined, nonencapsulated tumor with a patternless arrangement of alternating hypercellular and hypocellular regions of spindle cells against a collagenous background of variable vascularity. The tumors are richly vascular with many dilated vessels showing a staghorn appearance. ${ }^{3-6,11,23,27,28} \mathrm{Be}-$ cause of the variety of histopathologic components in SFT, it is necessary to choose complete excision of the mass rather than

fine-needle aspiration or excisional biopsy to avoid misdiagnosis. ${ }^{11}$ Moreover, recurrence and malignant transformation of orbital SFTs occur more often than in schwannomas. ${ }^{2,6,11-15}$ Therefore, it is important to differentiate SFTs from schwannomas and other orbital tumors before treatment recommendations are made.

Orbital schwannoma, constituting $1 \%$ of all orbital tumors, is more common than SFT. It is a peripheral nerve sheath tumor originating purely from Schwann cells. ${ }^{19,20}$ The pathologic feature of schwannoma is characteristic, with the tumor composed of Antoni A and Antoni B patterns in variable proportions. The Antoni A pattern appears solid, while the Antoni B pattern shows loose myxomatous tissue. ${ }^{12}$

Although pathologic features of SFT are different from those of schwannoma, orbital SFT and schwannoma show similar clinical findings, making differential diagnosis difficult in clinical practice. . $3,8,11,19,28$ Therefore, a noninvasive method to distinguish SFT from schwannoma in the orbit could be clinically useful, especially in cases in which biopsy would be particularly difficult or the patient is not a good candidate for surgical resection.

Many lesions present as round or ovoid masses in the orbit, notably cavernous hemangioma, schwannoma, pleomorphic adenoma of the lacrimal gland, dermoid cyst, and SFT of the orbit. MR imaging can readily distinguish some of these tumors on the basis of characteristic imaging findings. ${ }^{18,19,22}$ Cavernous hemangioma, for example, is characterized by "progressive enhancement over time." 19 Pleomorphic adenoma is generally centered in 

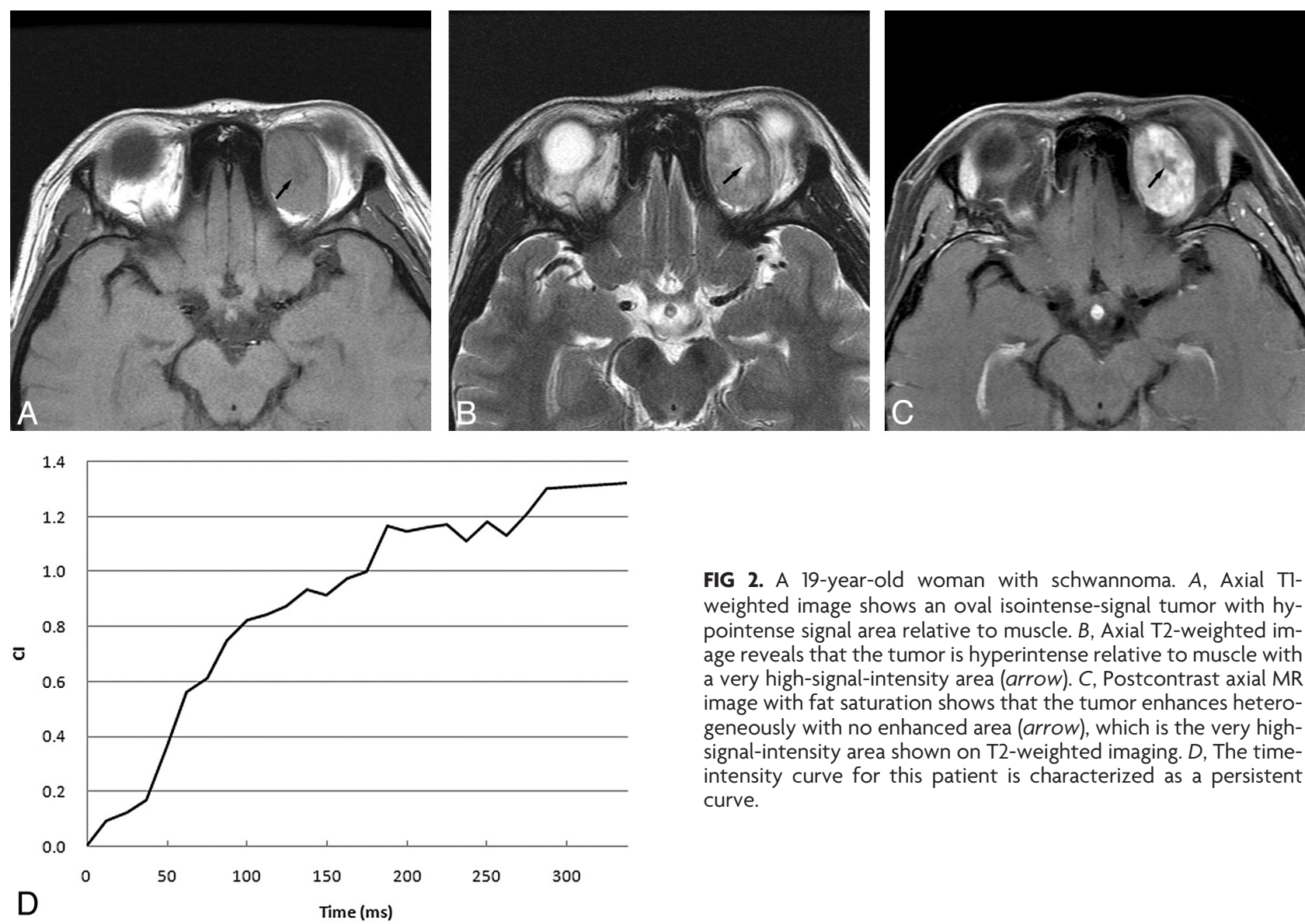

FIG 2. A 19-year-old woman with schwannoma. A, Axial T1weighted image shows an oval isointense-signal tumor with hypointense signal area relative to muscle. $B$, Axial T2-weighted image reveals that the tumor is hyperintense relative to muscle with a very high-signal-intensity area (arrow). C, Postcontrast axial MR image with fat saturation shows that the tumor enhances heterogeneously with no enhanced area (arrow), which is the very highsignal-intensity area shown on T2-weighted imaging. $D$, The timeintensity curve for this patient is characterized as a persistent curve.

the lacrimal gland and is well-circumscribed and T2 hyperintense. ${ }^{29}$ T1 hyperintensity and a lack of enhancement on MR imaging are characteristic findings of dermoid cyst. Precontrast MR imaging findings and an enhancement pattern of SFT in the orbit are so similar to those of schwannoma, however, that orbital SFT is often misdiagnosed as schwannoma preoperatively. ${ }^{8,9}$

Our results suggested that the type of TIC on DCE MR imaging and the enhancement pattern of very high-signal-intensity areas shown on T2-weighted imaging might be useful in the differentiation between SFT and schwannoma in the orbit.

On DCE MR imaging in our study, all 9 SFTs showed a washout curve, which has usually been reported in malignant tumors. ${ }^{21,30}$ Most schwannomas, however, displayed persistent or plateau-shaped curves. Kim et $\mathrm{al}^{2}$ reported that 3 patients with orbital SFT showed rapid enhancement with early washout of contrast material in the tumors on dual-phase CT. The period of enhancement in our 9 orbital SFTs on DCE MR imaging was relatively longer than that on dual-phase CT, probably because MR imaging enhancement is more sensitive than CT in demonstrating the vascularity of this tumor. The washout of contrast medium from tumors could be indicated by the washout ratio, which correlated with the fibrous-versus-cellular stroma in the tumors. ${ }^{30}$ High-fibrous and low-cellularity stroma would have a low washout ratio, while low-fibrous and high-cellularity stroma would show a high washout ratio. Because of high-cellularity stroma in the tumor, orbital SFT demonstrated a washout curve. The persistent or plateau-shaped curves shown in orbital schwan-

noma may be the result of the loose arrangement of cellularity in the tumor.

We found that the degree of enhancement of the very highsignal-intensity areas shown on T2-weighted imaging in orbital SFT was different from that in orbital schwannoma because most areas enhanced markedly, while in schwannoma, the areas generally showed no enhancement. This different enhancement pattern of the very high-signal-intensity areas in the tumors might be helpful in the differential diagnosis. It had been reported that the higher T2 signal intensity areas in schwannoma are related to thrombosis of degenerating blood vessels, hemorrhage, hyaline degeneration, and/or microcystic foci in Antoni B pattern, which could explain the lack of enhancement of these areas. ${ }^{9}$ The markedly enhanced very high T2 signal-intensity areas in orbital SFT might be related to areas of various cellular components rather than to the cystic or necrotic foci seen in schwannoma.

Although there was a statistical difference between orbital SFT and schwannoma in location and T2 signal intensity in our study, we found that many cases of both diseases may occur in the same location and show the same T2 signal intensity. Therefore we think that location and T2 signal intensity are nonspecific in differential diagnosis.

\section{CONCLUSIONS}

MR imaging can be useful in differentiating orbital SFT and schwannoma. We identified a statistically significant difference in the enhancement pattern of the very high-signal-intensity areas 
shown on T2-weighted imaging and the type of TICs, characteristics that could help differentiate SFT from schwannoma in the orbit.

\section{ACKNOWLEDGMENTS}

We thank Dr Li Wei of the Department of Pathology, Beijing Tongren Hospital, for preparing pathologic data, and Dr Feng Li of the Department of Radiology, University of Chicago, for useful suggestions in this study.

\section{REFERENCES}

1. Furusato E, Valenzuela IA, Fanburg-Smith JC, et al. Orbital solitary fibrous tumor: encompassing terminology for hemangiopericytoma, giant cell angiofibroma, and fibrous histiocytoma of the orbit: reappraisal of 41 cases. Hum Path 2011;42:120-28

2. Kim HJ, Kim H-J, Kim YD, et al. Solitary fibrous tumor of the orbit: CT and MR imaging findings. AJNR Am J Neuroradiol 2008;29: 857-62

3. Leoncini G, Maio V, Puccioni M, et al. Orbital solitary fibrous tumor: a case report and review of the literature. Pathol Oncol Res 2008;14:213-17

4. Goldsmith JD, Rijn M, Syed N. Orbital hemangiopericytoma and solitary fibrous tumor: a morphologic continuum. Int J Surg Pathol 2001;9:295-302

5. Cerdá-Nicolás M, Lopez-Gines C, Gil-Benso R, et al. Solitary fibrous tumor of the orbit: morphological, cytogenetic and molecular features. Neuropathology 2006;26:557-63

6. Bernardini FP, de Conciliis C, Schneider S, et al. Solitary fibrous tumor of the orbit: is it rare? Report of a case series and review of the literature. Ophthalmology 2003;110:1442-48

7. Westra WH, Gerald WL, Rosai J. Solitary fibrous tumor: consistent CD34 immunoreactivity and occurrence in the orbit. Am J Surg Pathol 1994;18:992-98

8. Kim HJ, Lee HK, Seo JJ, et al. MR imaging of solitary fibrous tumors in the head and neck. Korean J Radiol 2005;6:136-42

9. Savino G, Aliberti S, Colucci D, et al. Atypical presentation of a case of solitary fibrous tumor of the orbit. Orbit 2009;28:176-78

10. Kashyap S, Pushker N, Meel, R, et al. Orbital schwannoma with cystic degeneration. Clin Experiment Ophthalmol 2009;37:293-98

11. Krishnakumar S, Subramanian N, Mohan ER, et al. Solitary fibrous tumor of the orbit: a clinicopathologic study of six cases with review of the literature. Surv Ophthalmol 2003;48:544-54

12. Gündüz K, Shields CL, Gunalp I, et al. Orbital schwannoma: corre- lation of magnetic resonance imaging and pathology findings. Graefes Arch Clin Exp Ophthalmol 2003;241:593-97

13. Wang Y, Xiao LH. Orbital schwannomas: findings from magnetic resonance imaging in 62 cases. Eye 2008;22:1034-39

14. Girnita L, Sahlin S, Orrego A, et al. Malignant solitary fibrous tumour of the orbit. Acta Ophthalmol 2009;87:464-67

15. Tam ES, Chen EC, Nijhawan N, et al. Solitary fibrous tumor of the orbit: a case series. Orbit 2008;27:426-31

16. Johnson TE, Onofrey CB, Ehlies FJ. Echography as a useful adjunct in the diagnosis of orbital solitary fibrous tumor. Ophthal Plast Reconstr Surg 2003;19:68-74

17. Garcia-Bennett J, Olivé CS, Rivas A, et al. Soft tissue solitary fibrous tumor: imaging findings in a series of nine cases. Skeletal Radiol 2012;41:1427-33

18. Lemke A, Kazi I, Felix R. Magnetic resonance imaging of orbital tumors. Eur Radiol 2006;16:2207-19

19. Xian J, Zhang Z, Wang Z, et al. Evaluation of MR imaging findings differentiating cavernous haemangiomas from schwannomas in the orbit. Eur Radiol 2010;20:2221-28

20. Abe T, Kawamura N, Homma H, et al. MRI of orbital schwannomas. Neuroradiology 2000;42:466-68

21. Xian J, Zhang Z, Wang Z, et al. Value of MR imaging in the differentiation of benign and malignant orbital tumors in adults. Euro Radiol 2010;20:1692-702

22. Goha P, Gi M, Charlton A, et al. Review of orbital imaging. Eur J Radiol 2008;66:387-95

23. Ganly I, Patel SG, Stambuk HE, et al. Solitary fibrous tumors of the head and neck: a clinicopathologic and radiologic review. Arch Otolaryngol Head Neck Surg 2006;132:517-25

24. Motoori K, Hanazawa T, Yamakami I, et al. Intra- and extracranial solitary fibrous tumor of the trigeminal nerve: $\mathrm{CT}$ and MR imaging appearance. AJNR Am J Neuroradiol 2010;31:280-81

25. Wakisaka N, Kondo S, Murono S, et al. A solitary fibrous tumor arising in the parapharyngeal space, with MRI and FDG-PET findings. Auris Nasus Larynx 2009;36:367-71

26. Robinson LA. Solitary fibrous tumor of the pleura. Cancer Control 2006;13:264-69

27. Gengler C, Guillou L. Solitary fibrous tumour and haemangiopericytoma: evolution of a concept. Histopathology 2006;48:63-74

28. O'Donovan DA, Bilbao JM, Fazl M, et al. Solitary fibrous tumor of the orbit. J Craniofac Surg 2002;13:641-44

29. Mafee M, Edward DP, Koeller KK, et al. Lacrimal gland tumors and simulating lesions: clinicopathologic and MR imaging features. $R a$ diol Clin North Am 1999;37:219-39, xii

30. Yabuuchi H, Fukuya T, Hachitanda Y, et al. Salivary gland tumors: diagnostic value of gadolinium-enhanced dynamic MR imaging with histopathologic correlation. Radiology 2003;226:345-54 